\title{
Coupling running through the Looking-Glass of dimensional Reduction
}

\author{
D.V. Shirkov \\ Bogoliubov Lab., JINR, Dubna
}

\begin{abstract}
The dimensional reduction, in a form of transition from four to two dimensions, was used in the 90s in a context of HE Regge scattering.Recently, it got a new impetus in quantum gravity where it opens the way to renormalizability and finite short-distance behavior.

We consider a QFT model $g \varphi^{4}$ with running coupling defined in both the two domains of different dimensionality; the $\bar{g}\left(Q^{2}\right)$ evolutions being duly correlated at the reduction scale $Q \sim M$. Beyond this scale, in the deep UV 2-dim region, the running coupling does not increase any more. Instead, it slightly decreases and tends to a finite value $\bar{g}_{2}(\infty)<\bar{g}_{2}\left(M^{2}\right)$ from above.

As a result, the global evolution picture looks quite peculiar and propose a base for the modified scenario of gauge couplings behavior with UV fixed points provided by dimensional reduction instead of leptoquarks.
\end{abstract}

\section{Introduction}

\subsection{Motivation}

Initial motive for considering the topic mentioned in the title is related to the hot quest of the Higgs particle still escaping from direct observation. Anticipating the possibility that no Higgs peak will be observed in the "window" $140 \pm 25 \mathrm{GeV}$, we try to investigate the Ginzburg-Landau-Higgs possibility, with Higgs field being a classical field with nonzero constant component $\sim 250 \mathrm{GeV}$ sufficient for the mass production in the current version of SM. In other words, we are inclined to use the fact that the Higgs mechanism for creating masses could work when the Higgs field is not a quantum one being rather an analog of the Ginzburg-Landau two-component order parameter from the theory of superconductivity 1 .

However, changing a quantum Higgs for the classical external field yields the trouble of renormalization in the EW sector of SM. Having no intention to enter this complicated problem we prefer to postpone its solution and look for some temporary

\footnotetext{
${ }^{1}$ For details see our recent overview [1]
} 
practical remedy for the time being 2 . The possible way is to involve an invariant regularization procedure, like the Pauli-Villars one. To this goal, it is more intriguing to exploit a transition from the four-dimensional manifold to the one with a smaller number of dimensions $d<4$ at high enough energy or small distance. Technically, this could provide us with artificial cutoff with only one additional parameter, the range of reduction.

The trick with changing the number of dimensions is a frequent one in current literature (on superstrings ect.) devoted to the HE behavior. This transition a lá Kaluza-Klein to a larger number of dimensions $D>4$ inevitably confronts us with the non-renormalizability. Instead, we consider another, a rather opposite possibility, the dimensional reduction (DR).

To explore some practical aspects of the DR, as a first test-flight to terra incognita, we turn here to a limited subject, the issue of transferring the renormalization-invariant running coupling $\bar{g}\left(Q^{2}\right)$ through the region of reduction and relating its behavior in two domains with different dimensionality.

\subsection{Dimensional reduction.}

The dimensional reduction was used first about 15 years agd 3 as a pragmatic tool in the analysis of the HE Regge scattering. This line of reasoning was refreshed 4] quite recently in the context of the LHC physics with a more explicit emphasis of the DR physical implementation. In the last decade it became quite popular in quantum gravity. Here, a class of models has been devised by Horava (see, e.g., paper [5] and references therein) with asymptotic anisotropy between space and time dimensions in the short distance UV limit 4 .

Our attitude do not imply any modification of the special relativistic concept of the time. We just have in mind some smooth reduction of the spatial topological dimensions. Probably this scenario is akin one another approach formulated recently 8 for the quantum field living in the fractal spacetime.

Agreement on DR. One can discuss the mechanism of dimensional reduction either in the space-time terms or in the energy-momentum ones using the presumptive assumption that

Reduction at the space-time scale $x_{d r} \sim 1 / M_{d r}$ is, in a sense, equivalent to the reduction at the energy-momentum scale $p_{d r} \sim M_{d r}$.

\footnotetext{
${ }^{2}$ Here, on can rely upon the well-known examples :

- In the early 30s, Dirac was brave enough 2 to use cutoff on the proton mass in discussing the momentum dependence of electron charge $e(Q)$, a prototype of the QED running coupling.

- In the 50s and 60s, after the devising of the renormalization procedure, phenomenologists still widely and fruitfully used hardly non-renormalizable 4-fermion interaction à lá Fermi for analyzing weak interactions. They postponed the problem solution till reaching the so called "unitary limit" at $W_{\text {c.m. }} \sim 100 \mathrm{GeV}$.

${ }^{3}$ See paper 3 and references therein.

${ }^{4}$ This activity was motivated by remarkable observation by Ambjorn, Loll and others $[\underline{6}$ in the causal dynamical triangulation approach to quantum gravity on lattice. See also an overview 7 .
} 
This tentative agreement will be used below to compare the Lagrangian (spacetime) approach with the direct ad hoc modification of the momentum integration of Feynman integrals. In the course of the first approach we use a sharp conjunction as an approximation to a softer mechanism of a continuous DR in the second one.

Classical illustration. To illustrate the idea of approximation, imagine a wine bottle (e.g., posed vertically) like one presented on Fig.1(a). It consists of the main cylindrical body $B$ with a relatively large radius $R$ and a length $L$. The bottle's neck $N$ of length $l$ and smaller radius $r$ is connected with the main part by a "collar" - a narrowing transition region $C$ of a varying radius and a short length $l_{\text {coll }}$.

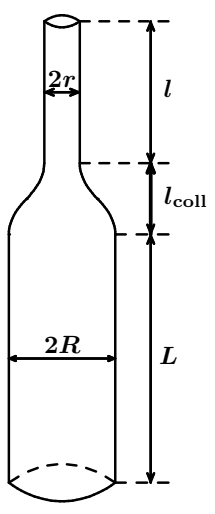

(a)

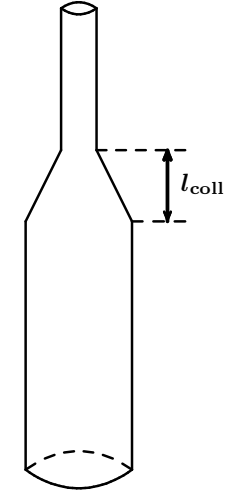

(b)

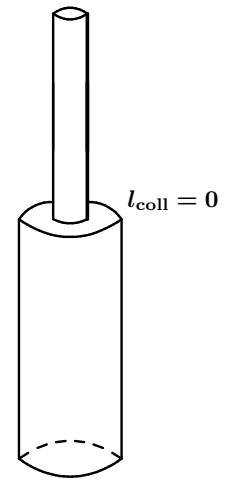

(c)

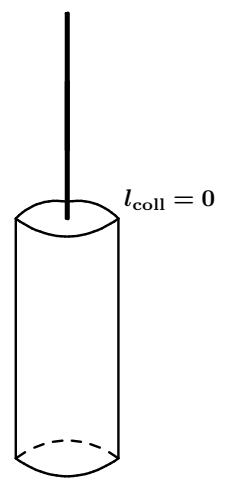

(d)

Figure 1:

(a)- Wine-bottle with a long body and a long neck; (b) - Bottle with a conical collar:

(c) - Bottle with a horizontal collar: (d) - Bottle with a horizontal collar and a thread-like neck.

Suppose now that there is a mathematical model for some process on the twodimensional surface $S_{2}$ of the bottle. One can have in mind a stationary boundary value problem for the second order partial differential equation(s), or some dynamical process like wave propagation with radiation or heat conductivity and so on.

A number of problems with exact analytical solutions on the surfaces $S_{R, L}$ and $S_{r, l}$ of cylindrical parts can be found. Many of them could be solved for the whole two-dimensional manifold $S_{2}=S_{R, L}+S_{r, l}+S_{\text {coll }}$ for simple enough smooth forms of the junction collar region $C$.

Of particular interest are non-stationary processes, like a solitary wave propagating upward due to some short-time perturbation at the lower edge of the bottom.

It will be instructive to study several issues :

- the dependence of solution details on the form of the surface $S_{\text {coll }}$ of the collar region; - transition to a sharp change of radius (to a horizontal collar) as $l_{\text {coll }} \rightarrow 0$, Fig. 1(c); - the limiting case $r \rightarrow 0$, that is a transition from the 2-dim surface of the neck $S_{r, l}$ to the 1-dim linear manifold $S_{0, l} \rightarrow L_{l}$, Fig. 1(d).

In the further analysis, along with smooth transition in Section 2.1, we shall use "hard conjunction" in Section 2.2 of two regions with different dimensions. The first 
one resembles the Fig.1(a) in the limit $r \rightarrow 0$, while the second one is an analog of a system presented on Fig.1(d).

\section{Effective $\bar{g}$ for the $\varphi^{4}$ model in various dimensions}

Take the one-component scalar massive quantum field $\varphi(x)$ with the self-interaction Lagrangian

$$
L=T-V ; \quad V(m, g ; \varphi)=\frac{m^{2}}{2} \varphi^{2}+\frac{4 \pi^{d / 2} M^{4-d}}{9} g_{d} \varphi^{4} ; \quad g>0
$$

in parallel in four $(d=4)$ and two $(d=2)$ dimensions.

Limit ourselves to the one-loop approximation level for $\bar{g}$ that corresponds to the only Feynman diagram contribution, the first correction to the 4-vertex function, Fig.2.

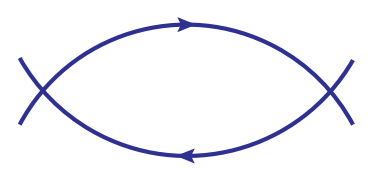

Figure 2: One-loop vertex diagram

Its contribution $I$ enters into the effective running coupling as follows:

$$
\bar{g}\left(q^{2}\right)=\frac{g_{i}}{1-g_{i} I\left(q^{2} ; m^{2}, m_{i}^{2}\right)} .
$$

\subsection{Smooth DR in the momentum picture}

We start with reduction of dimensions in Feynman integral by modifying metrics in the momentum space

$$
d k=d^{4} k \rightarrow d_{M} k=\frac{d^{4} k}{1+k^{2} / M^{2}} ; \quad k^{2}=\mathbf{k}^{2}-k_{0}^{2} .
$$

In particular, for the one-loop integral Fig.2 one gets

$$
I\left(\frac{q^{2}}{m^{2}}\right)=\frac{i}{\pi^{2}} \int \frac{d k}{\left(m^{2}+k^{2}\right)\left[m^{2}+(k+q)^{2}\right]} \rightarrow \frac{i}{\pi^{2}} \int \frac{d_{M} k}{\left(m^{2}+k^{2}\right)\left[m^{2}+(k+q)^{2}\right]}=J(\kappa ; \mu),
$$

with $\kappa=q^{2} / 4 m^{2}, \mu=M^{2} / m^{2}, q^{2}=\mathbf{q}^{2}-q_{0}^{2}$. The integral $J$ can be calculated explicitly. We give its asymptotics. In the "deep 4-dim" region $m^{2} \ll q^{2} \ll M^{2}$ one gets an "intermediate" logarithmic behavior with the $M$ playing the role of the Pauli-Villars regulator. Meanwhile, in the "deep 2-dim" region $q^{2} \gg M^{2} \gg m^{2}$, the UV limit is finite. In usual normalization

$$
J \rightarrow J_{i}=J\left(q^{2} / 4 m^{2} ; \mu\right)-J\left(m_{i}^{2} / 4 m^{2} ; \mu\right) ; \quad m_{i} \sim m,
$$


one has

$$
J_{i}^{[4]}(\kappa ; \mu) \sim \ln \left(\frac{q^{2}}{m_{i}^{2}}\right) ; \quad J_{i}^{[2]}(\kappa ; \mu) \sim \ln \left(\frac{4 M^{2}}{m_{i}^{2}}\right)+\frac{M^{2}}{q^{2}} \ln \frac{q^{2}}{M^{2}} .
$$

The first expression is rising; while the second, decreasing. The maximum value of $J$ is attained at the DR scale $q^{2} \sim M^{2}$ and is close to $\ln \left(M^{2} / m^{2}\right)$. Hence, due to the DR, the $\bar{g}\left(q^{2}\right)$ evolution changes drastically. The effective coupling slightly diminishes 5 beyond the reduction scale and tends to a finite value, see Fig.3.

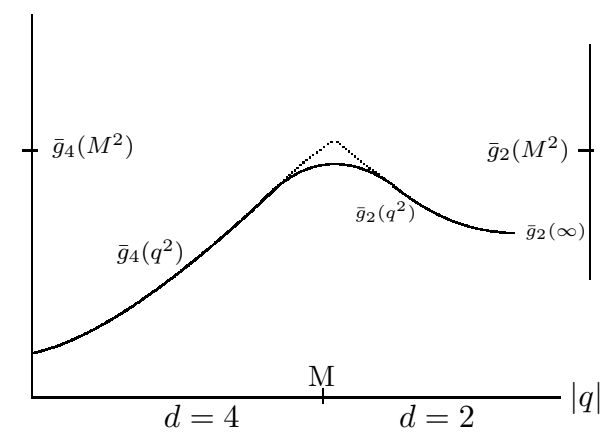

Figure 3: The effective coupling evolution for the $\varphi^{4}$ model with DR.

\subsection{Reduction with Lagrangians}

Now, along with the Agreement on $D R$, return to the Lagrangian description eq.(1) in terms of fields. There, under transition to the $d=2$ case, the field $\varphi_{4}(x)$ loses its dimensionality $\varphi_{4}(x) \rightarrow \varphi_{2}(x) \sim M^{-1} \varphi_{4}(x)$, while the coupling constant (as in eq.(1)) acquires it: $g_{4} \rightarrow M^{2} g_{2}$, with some parameter $M$ that we put equal to the DR scale $M=M_{d r}$. Below, the dimensionless constant $g_{2}$ will be used.

\subsubsection{Invariant coupling in $d=2$.}

In two dimensions, one can as well use finite Dyson transformations, formulate RG invariance and mass-dependent renormalization group6, define a "massive" running coupling with its explicit one-loop solution [13] (see, also $§ 43.1$ in [10] 7 and paper [14])

$$
\bar{g}^{[2]}\left(q^{2}\right)=\frac{g}{1-g M^{2} I_{2}\left(q^{2} / m^{2}\right)}=\frac{g}{1-g J_{2}\left(q^{2} / m^{2}\right)} ; J_{2}\left(\frac{q^{2}}{m^{2}}\right)=\frac{i}{\pi} \int \frac{M^{2} d^{2} k}{\left(m^{2}+k^{2}\right)\left[m^{2}+(k+q)^{2}\right]} .
$$

Here, $J_{2}$ is a finite one-loop contribution from the 4-vertex diagram, Fig.2, in two dimensions. It is a positive monotonously decreasing function. Asymptotically, $J_{2} \sim$ $\left(M^{2}\right) / q^{2} \ln \left(q^{2} / m^{2}\right)$, - just like in the second eq.(44). Therefore, two-dimensional effective coupling in the UV limit tends to its limiting fixed value from above.

\footnotetext{
${ }^{5}$ This reverse evolution is not seen in the common massless, pure logarithmic, RG analysis.

${ }^{6}$ As it was introduced in the mid-50s in [9]: see also Chapter VIII in monograph [10] or Chapter IX, $\S 51$ in its 3rd edition [11] and Appendix 9 in the textbook [12].

${ }^{7}$ Unhappily, this piece was omitted in the next edition [11].
} 


\subsubsection{Hard conjunction at the reduction scale}

To obtain the joint picture of coupling evolution, one has to consider a transition from the "low-energy" 4-dim region $q^{2}<M^{2}$ to the "high-energy" 2-dim one $q^{2}>M^{2}$.

For the "hard" conjunctions, the continuity property $\bar{g}_{4}\left(M^{2}\right)=\bar{g}_{2}\left(M^{2}\right)=g_{M}$ yields

$$
\bar{g}_{4}\left(q^{2}\right)=\frac{g_{M}}{1-g_{M} \ln \left(q^{2} / M^{2}\right)} ; \quad q^{2} \leq M^{2}
$$

and, along with expression (5),

$$
\bar{g}_{2}\left(q^{2}\right)=\frac{g_{M}}{1-g_{M}\left[J_{2}\left(q^{2} / m^{2}\right)-J_{2}\left(M^{2} / m^{2}\right)\right]} ; \quad q^{2} \geq M^{2}
$$

with finite UV limit

$$
\bar{g}_{2}(\infty)=\frac{g_{M}}{1+g_{M} J_{2}\left(M^{2} / m^{2}\right)}<g_{M}
$$

This means that above the reduction scale the effective coupling evolves down to its final UV limit. This behavior corresponds to Fig.3.

\section{Discussion}

In the above analysis, one more alternative to the standard Higgs mechanism within the Standard Model was considered. The main idea consists in employing the possibility of reducing the number of dimensions in the far UV limit, the possibility that is intensively discussed now in the context of quantum gravity.

Taking for definiteness the reduction from common four dimensions to two dimensions at some high enough scale $|q| \sim M$, we studied the issue of effective coupling behavior for the $\varphi^{4}$ scalar model (1). Using hard conjunction, by continuity: $\bar{g}_{4}\left(M^{2}\right)=\bar{g}_{2}\left(M^{2}\right)$ just at the DR scale, we got the joint picture which is very close to the $\bar{g}$ behavior in the case of continuous DR by changing metric in the momentum space via eq.(3). The resulting picture is presented on Fig.3.

Its essential technical feature is the change of the familiar logarithmic growth of the $\bar{g}_{4}$ running coupling for the slight decrease of the $\bar{g}_{2}\left(Q^{2}\right)$ beyond the reduction scale. There, in the effective 2-dim region, the $\varphi_{2}^{4}$ interaction is super-renormalizable and the one-loop mass-dependent contribution to running coupling is a decreasing function.

Due to this, the $\bar{g}\left(Q^{2}\right)$ evolution changes its pattern. At the infinity it tends to its limiting value from above, as in eq.(7).

This peculiar property, the reverse running coupling evolution to a fixed point beyond the "distorted mirror" marked by the reduction scale, has a chance to upgrade the Grand Unification scenario with the $M$ value being of the order or even greater than the hypothetical lepto-quark scale - see qualitative illustration on the Fig.4.

Indeed, a rough estimate reveals that difference between $1 / \bar{g}(\infty)$ and $1 / g_{M}$ is of order of unity; being imported to the GUT context, it could produce effect numerically sufficient to close the famous discrepancy triangle[15].

\footnotetext{
${ }^{8}$ In the classical wine-bottle model, this hard conjunction corresponds to Fig.1(d).
} 


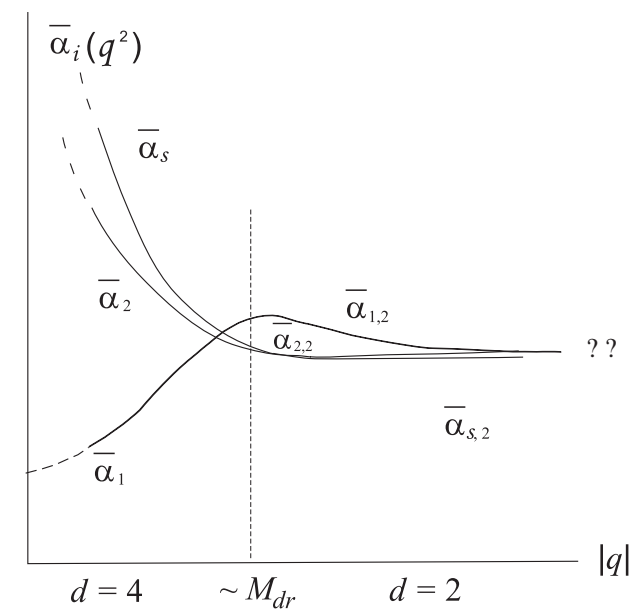

Figure 4: Modified scheme of the hypothetical Great Unification provided by dimensional reduction instead of leptoquarks.

The notable observation is that the change of geometry could yield the same final result as an explicit change of dynamics (by adding leptoquark fields etc.).

Among further quests that are in order, let us put in the first place the issue of examining the chance of detecting some physical signal "through the looking-glass at scale $M$ " that would provide us with direct evidence on the existence of dimension reduction of any kind. It could be fruitful to study some classical problems formulated at the end of Section 1 to enrich our intuition.

\section{Acknowledgments}

It is a pleasure to thank Drs. Irina Aref'eva and Plamen Fiziev for useful discussions. The courtesy of Dr. Jean Zinn-Justin for informing on relevant literature is also appreciated. This research has been partially supported by the presidential grant Scientific School-3810.2010.2 and by RFFI grant 08-01-00686.

Few relevant integrals.

\section{Appendix}

1. First, an auxiliary one-loop Feynman integral (of the Fig.2 type) with different masses (see eq.(DVI.31) on page 341 of the textbook [12] American edition)

$$
f\left(q^{2}\right)=f\left(\frac{q^{2}}{M^{2}} ; \mu^{2}\right) \sim \frac{i}{\pi^{2}} \int \frac{d^{4} k}{\left(m^{2}+k^{2}\right)\left(M^{2}+(k+q)^{2}\right)} ; \quad q^{2}=\mathbf{q}^{2}-q_{0}^{2} .
$$

According to eq.(DVI.32), at $\mu^{2}=m^{2} / M^{2} \ll 1$ the result of integration subtracted at zero, $f_{0}(0)=0$ looks like

$$
f_{0}\left(\frac{q^{2}}{M^{2}} ; \mu^{2}\right)=\frac{K(q)}{2} \ln \frac{M^{2}+q^{2}+q^{2} K}{M^{2}+q^{2}-q^{2} K}+\frac{1}{2}\left(1+\frac{M^{2}}{q^{2}}\right) \ln \frac{M^{2}}{m^{2}}-1+o\left(\frac{m^{2}}{M^{2}}\right) ;
$$

with

$$
K(q)=\left[\left(1+\frac{(M+m)^{2}}{q^{2}}\right)\left(1+\frac{(M-m)^{2}}{q^{2}}\right)\right]^{1 / 2} \sim 1+\frac{M^{2}}{q^{2}}-\frac{2 m^{2} M^{2}}{q^{2}\left(q^{2}+M^{2}\right)}+o\left(\frac{m^{2}}{M^{2}}\right) .
$$


Start first with intermediate logarithmic asymptotics at $m^{2} \ll q^{2} \ll M^{2}$. There,

$$
f_{0}^{U V}\left(\frac{q^{2}}{M^{2}}\right) \simeq\left(1+\frac{M^{2}}{q^{2}}\right) \ln \frac{M^{2}+q^{2}}{M^{2}}-1+o\left(\frac{m^{2}}{q^{2}}\right) .
$$

This asymptotic expression still satisfies the normalization condition $f_{0}^{U V}(0)=0$.

2. Another 4-dim integral in the dimensionless normalization

$$
J(\kappa ; \mu)=\frac{i}{\pi^{2}} \int \frac{M^{2} d k}{\left(m^{2}+k^{2}\right)\left[m^{2}+(k+q)^{2}\right]\left(M^{2}+k^{2}\right)}
$$

can be presented via $f_{0}$ in the simple form

$$
J(\kappa ; \mu)=\frac{M^{2}}{M^{2}-m^{2}}\left[f_{o}\left(\frac{q^{2}}{m^{2}} ; 1\right)-f_{o}\left(\frac{q^{2}}{M^{2}} ; \mu^{2}\right)\right] .
$$

It is evident that at the "D4 asymptotic region" $m^{2} \ll q^{2} \ll M^{2}$ the first term $f_{0}\left(q^{2} / m^{2}\right)$ with its rising logarithmic asymptotics dominates, while beyond the looking glass in the D2 $\mathrm{UV}$ region $q^{2} \gg M^{2}$ the whole expression tends to finite limit.

3. For the very end, one more expression resulting from the 2-dim integral (5) in the Euclidean domain at $q^{2}>0$ and $k^{2}=k_{1}^{2}-k_{0}^{2} \rightarrow k_{E}^{2}=k_{1}^{2}+k_{4}^{2}>M^{2}$

$$
\begin{gathered}
J_{2}\left(\frac{q^{2}}{m^{2}}\right)=\frac{i}{\pi} \int_{M} \frac{M^{2} d^{2} k}{\left(m^{2}+k^{2}\right)\left[m^{2}+(k+q)^{2}\right]}=\frac{1}{\pi} \int_{M} \frac{M^{2} d_{E}^{2} k}{\left(m^{2}+k_{E}^{2}\right)\left[m^{2}+(k+q)_{E}^{2}\right]} ; \\
J_{2}(0)=\frac{M^{2}}{m^{2}+M^{2}} ; \quad J_{2}\left(\frac{M^{2}}{m^{2}}\right)=
\end{gathered}
$$

4. Numerical estimate. Due to eqs.(8),(13), the difference

$$
\frac{1}{\bar{g}(\infty)}-\frac{1}{g_{M}}=J_{2}\left(M^{2} / m^{2}\right)
$$

is of order of unity. Accordingly, being imported to the GUT context, it can produce effect numerically sufficient to close the famous discrepancy triangle.

\section{References}

[1] D.V.Shirkov, "60 years of Broken Symmetries in Quantum Physics", Phys.Usp. 52: 549557,2009; [physics.hist-ph]:0903.3194

[2] P.A.M. Dirac in Theorie du Positron (7-eme Conseil du Physique Solvay: Structure et propriete de noyaux atomiques, Oct.1933), Gauthier-Villars, Paris,1934, pp.203-230.

[3] I.Ya. Aref'eva, Phys.Lett., B 325 (1994) 171; hep-th/9311115.

[4] Luis Anchordoqui, et al., "Vanishing Dimensions and Planar Events at the LHC." [hepph:1003.5914]

[5] Petr Horava, Phys.Rev. D 79: 084008, (2009); hep-th:0901.3775; 
[6] J.Ambjorn, R. Loll, et al., "New aspects of two-dimensional quantum gravity", hepth:0911.4208; "Proper time is stochastic time in 2d quantum gravity", hep-th:0911.4211;

[7] M. Niedermaier, "The Asymptotic safety scenario in quantum gravity: An Introduction". Class.Quant.Grav. 24: R171,2007; gr-qc/0610018.

[8] Gianluca Calcagni, "Quantum field theory, gravity and cosmology in a fractal universe", JHEP 03(2010)120 [arXiv:hep-th/1001.0571].

[9] N.N.Bogoliubov and D.V.Shirkov, Nuovo.Cim. 3, 845-863 (1956).

[10] N.N. Bogoliubov and D.V.Shirkov, "Introduction to the Theory of Quantized Fields", Interscience, N.Y., 1959.

[11] N.N. Bogoliubov and D.V. Shirkov, "Introduction to the Theory of Quantized Fields", 3rd edition, Wiley-Interscience, N.Y., 1980.

[12] N.N. Bogoliubov and D.V. Shirkov, Quantum Fields, Benjamin/Cummings Publ., Reading, 1983.

[13] V.Z. Blank, D.V. Shirkov, Nucl.Phys. 2 356-370 (1956/57).

[14] D.V. Shirkov, Nucl.Phys. B 371 467-481 (1992).

[15] A.V.Gladyshev and D.I.Kazakov, "Supersymmetry and LHC", Phys.Atom.Nucl. 70 (2007) 1553-1567, e-Print: hep-ph/0606288. 